\title{
Enhancement of Systematic Design Processing by Diagrams
}

\author{
Buthayna Hasan Eilouti \\ Professor, Ph.D, Department of Architecture, Department of Interior Design Engineering, Prince Sultan \\ University, KSA. Buthayna@umich.edu
}

doi:10.5618/arch.2012.v1.n1.9 || Received: 23-04-2012, Accepted: 27-05-2012, Available online: 7-06-2012

\begin{abstract}
Diagrams play a significant role in most knowledge presentation areas. This is especially the case in disciplines concerned with conceptual design where data visualization, processing, documentation and presentation require graphic illustration to express their underlying tacit aspects. Despite their significance, the visual tools available in the toolbox of designers to help them apply generative processes are extremely limited. This paper introduces some conceptual and operative diagrams that aim to aid in the visualization of various stages of the design derivation and development processes. The structure underlying the proposed diagrams represents a basis for a diagrammatic framework for architectural designing that may help systematically induce form development, organize design sequence and externalize concept generative forces. The purpose of this framework is not to dictate a specific sequence of design, but rather to help designers process their designs in ways more structured than trial/error-based ones. The scope of the proposed framework includes all phases of designing that include the input, output, presentation and assessment activities, with a focus on the core design processing and form-making activities. To test the applicability of this framework, it was implemented to solve a real design problem in an architectural design studio. The feedback of the diagrammatic framework users was analyzed by a survey about its implementation in comparison to conventional design approaches. The results of this empirical study are reported and discussed. The results appear to support the role of diagrams in the enhancement of designing and learning processes. However, this contribution of diagrams to designing and learning needs further applications in various design settings to assess its limitations and strengths.
\end{abstract}

Keywords: Information visualization; Form architecture; Visual studies; Design conception; Operative diagram; Conceptual diagram; Design process

\section{Introduction: Diagrams in Design}

Diagrams are essential modes of representation in design communication, and tools of exploration in design derivation. They concisely convey problem interpretation, pre-design reasoning, problem solving, design conception, form evolution, design processing and product evaluation. They are widely used in most design disciplines, and most significantly in fields concerned with form making. The emphasis of this paper will be on architectural design.

Many diagramming strategies and applications are described in design and architectural literature. Some focused on specific aspects of diagramming such as the graphic representation of information and knowledge conceptualization [e.g. 1-7]. Some other research efforts were focused on representing the spatial syntax as well as the formal and topological relationships of design components [e.g. 8-9]. The psychological and intellectual attributes of diagramming were represented in some research topics as well [e.g. 10-12].Other research examples were more concerned with diagramming for reasoning [e.g. 13-14], and analytical diagramming for the concise encapsulation of information about contexts of design problems [e.g. 15]. However, diagram development as representative and operative tool and diagram-driven methods for conceptual designing did not receive enough attention of researchers. The focus here will be on developing diagrams that graphically and abstractly describe the forces, flows and sequences that a designer may consider before and during design processing. The diagrams developed in this paper, in turn, from a basis for a diagrammatic framework for design conception and processing that may employ basic diagrams to induce the design process.

Diagrams vary in their abstraction and symbolism according to the phase of design sequence they are applied in. They also vary according to many other contextual factors such as the cultural and experiential background of their senders and receivers. Diagrams as modes of communication significantly contribute to the practice and education of architectural design. For example, Alexander [16] describes a diagram as the starting point of design synthesis from which a design gradually evolves through matching design requirements to correspondent diagrams to eventually 
end up with a product that consolidates a tree of diagrams. In addition, Laseau [17] describes diagrams as abstract graphic language that consists of vocabulary and grammatical rules to work out problems and communicate solutions. Similarly, Lockard [18] argues that diagramming can be used to explore design solutions and to interact with visual information. Other examples of diagram contribution to design include their role in design creativity [e.g. 19], and their enhancement for design computing [e.g. 20].

Within the scope of this article, a diagram is understood as a form of data visualization that abstractly and graphically uses geometric elements (e.g. lines, nodes, circles, rectangles and arrows) and textual annotations to represent structure, hierarchy, enclosure and/or sequence of design products, as well as their related functions, processes and activities. It helps transform raw data into knowledge by applying metaphors that enable users to understand new concepts in terms of familiar experiences. As such, a diagram is basically a schematic drawing that may contain text, symbols, geometric shapes and directional arrows to describe conceptual, functional, spatial, organizational, hierarchical, topological, or/and sequential information. Its functions are typically iterative, experimental, cognitive and metaphorical, the framework of which embodies some epistemological as well as ontological concepts about design knowledge nature and building. Some of its major goals are to extract, reduce, and explore information. Other goals include the endeavor to organize, analyze and synthesize ideas; and to generate and manage design through visualizing complex relationships in simplified minimalist graphic forms.

Currently, there exist an extremely limited number of diagram types in the toolbox of architecture designers. These include bubble diagrams, functional relationship matrices, spatial organization flowcharts, and site analysis graphs. These are mostly devised for analytical purposes. The deficiency is even greater in the synthesis areas. Some efforts have been made to incorporate insights from other fields to encode the form assembly sequence in design. An example of these efforts is the spatial development of finite state automata for design process description [e.g. 21-23]. However, there is a continuous need for introducing new visual aids in design processing, presentation and visualization.

\section{Research Design}

New diagrams for design process and product visualization are proposed and presented in this paper. The research design consists of a theoretical research and an empirical study. The first is used to develop the proposed diagrams. The second is applied to test the applicability of the proposed diagrams in real design settings in general, and whether they can help novice design learners organize their processing techniques in particular.

The research assumptions include the ontological, the epistemological and the methodological assumptions. The first one is that design is the creative translation of imagination and knowledge into concrete components.

The concept assumed by the proposed diagrams in the second assumption is that design knowledge is acquired through a cognitive transformation of visual data representations.

The third assumption is that designers externalize their tacit knowledge through the implementation of diagrammatic approaches to designing.

The diagrams proposed in this paper may function as both operative and figurative aids. They aim to help guide the actual process of designing in the former type and describe data succinctly and visually in the latter. It is hypothesized here that a diagram-aided approach to designing improves design learning as well as design knowledge building, implementation, representation and evaluation.

\section{Diagrams for the Representation of Design Processing}

In general, design can be developed through five major inter-related, recursive and iterative phases. These are 1) the data input and reasoning phase, 2) the design development and processing phase, 3) the designing output production phase, 4) the product representation and documentation phase and finally 5) the product performance assessment phase. The five phases overlap as illustrated in Fig. 1 (a). The diagram shown in Fig. 1 (a) illustrates how the five phases are dependent on each other during both the forward cycle of product evolution and backward cycle of product evaluation. The functions of each are based on the previous, but they are combined in cycles of revisions and fine tuning. Within the process of designing, the pre-design input sub-systems enhance the design development and production subsystems which, in turn, are necessary to conduct design documentation and post-design performance simulation assessment. Similarly, a post-design evaluation of a product helps to inform a pre-design reasoning for a new design problem and possible solutions. Although activities of each phase represent prerequisite of the next, tasks within them may overlap. In other words, a designer does not have to wait for a phase to end in order to start the next. S/he may find part of the reasoning inspiring for a design concept, evaluate it and go back to explore more layers of the problem interpretation and solution. The diagram shown in (Fig. 1, b) demonstrates an example of one of the subsystems, 
that is the pre-design data input and information reasoning phase. This phase of design processing itself most likely starts with the data search, location, collection, preparation, investigation, classification, interpretation and representation. This analytical phase includes functional programming, site, clients and other design stakeholders input reasoning, context study, and case study analysis (Fig. 1, b). In the programming phase, the design program is developed. Its components and their space, finishing, furniture, equipment, lighting and acoustic requirements are planned. In the site and context analysis, the site and its surroundings are analyzed according to their spatial, environmental, social, cultural and natural forces. In the case study analysis, previous design solutions that are related partially or totally to the problem at hand are studied and relevant information are extracted from them as needed. In the stakeholders' input analysis, the requirements set by the clients and other people involved in the project at hand are considered and analyzed.

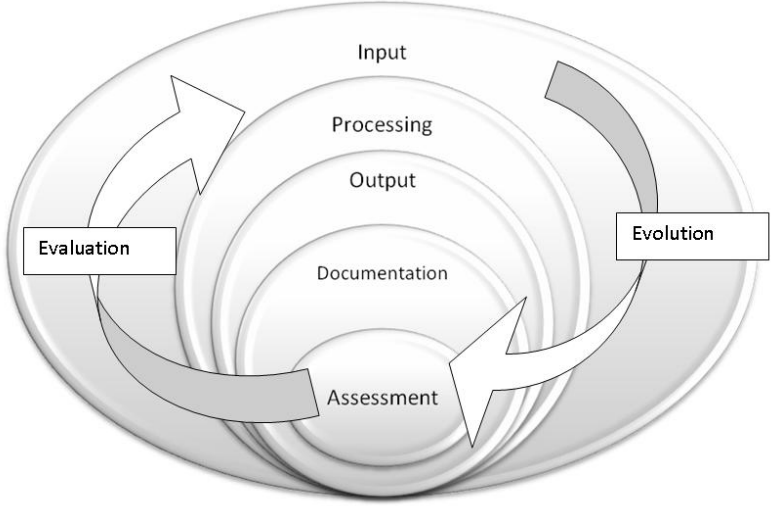

a

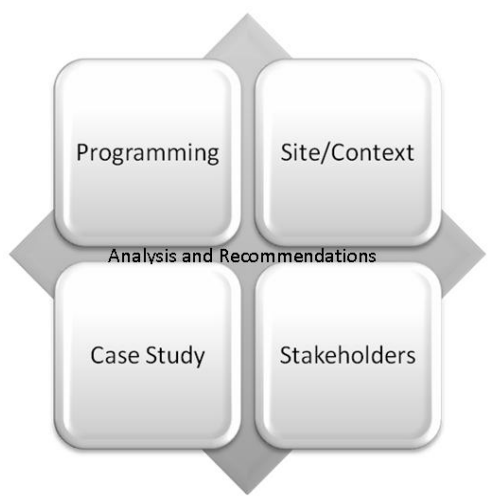

b

Figure 1. a: Design Processing Activities, b: Pre-Design Reasoning Activities.

4. The Designing Loops. The designing cycle is further demonstrated by Fig. 2. Its activities start with the knowledge management stage, where data collection, filtering, organization, analysis and transformation into meaningful knowledge take place. In this phase, the client's and other stakeholders' input is most important. The tasks of this phase include, as illustrated in Fig. 1(b), programming, case-study analysis, contextual studies and input from potential stakeholders. The next phase in the process is design where formal, functional and contextual requirements are considered and consolidated into a physical product. The third phase is product evaluation where physical and nonphysical environments are examined to assess user experience, comfort level and code compliance. The major player in the third stage is external examiners, project client, potential users or the designer him/herself who evaluates the design and provides feedback that may revise the whole design process. While the client deals with the input side, the evaluator with the output side, the designer is the main processor whose role moves between all players. The keyword of the first phase is information. It is the major axis about which pre-design knowledge management activities are centered. The keyword of the second phase is creativity which controls all the major activities of design generation. The keyword of the third phase is quality assurance where the major axis is about testing the main tangible and intangible attributes of the product and how they achieve a satisfactory quality level. None of the phases is deterministic and no clear demarcation can be identified between them as they are all intertwined. Following is a discussion for diagrams in each of the three phases.

\subsection{Diagrams for Design Knowledge Management.}

The knowledge management and reasoning phase is further illustrated in Fig. 3. As this figure shows, in the programming stage, the functional components of the design at hand are identified and their spatial and environmental control requirements are analyzed. The inter-relationships among the components are investigated and classified according to their strengths. Then, the components' topological relations are outlined and represented as a proportional bubble diagram or other easy to comprehend formats. Depending on the spatial grouping recommendations that may result from the bubble diagram analysis, program components can be clustered into major functional or spatial groups, which can be represented as an abstract zoning diagram. The result of this part of the analysis and development is the generation of a "functional solution" that is solely based on mapping a solution to the program requirements. The generated solution is context-free. It is a functional template that needs further customizations to fit in specific location and context. 


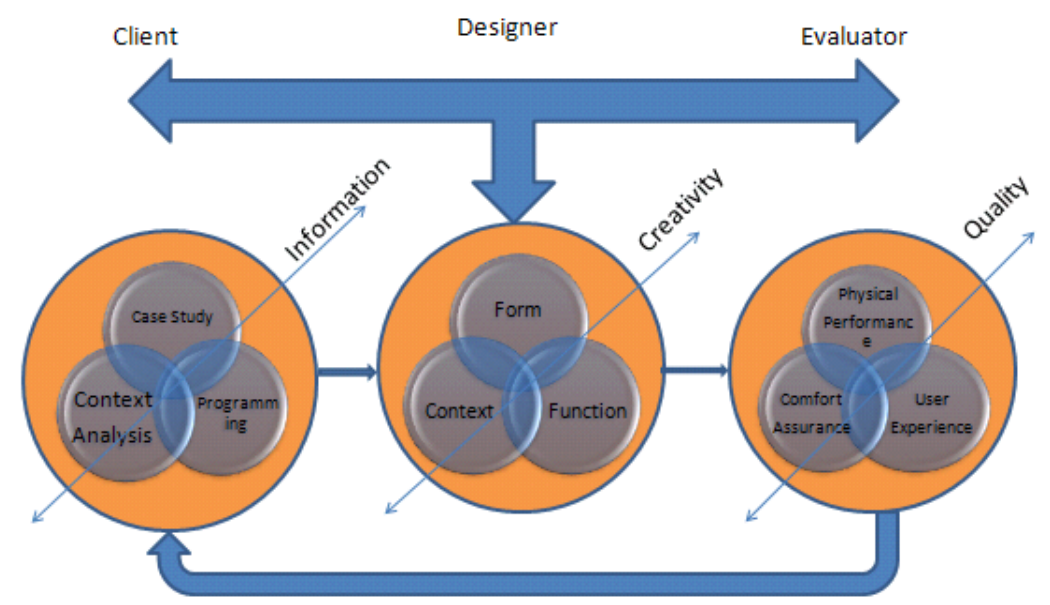

Precedent Analysis

Design Processing

Product Assesment

Figure 2. Design Processing Cycle.

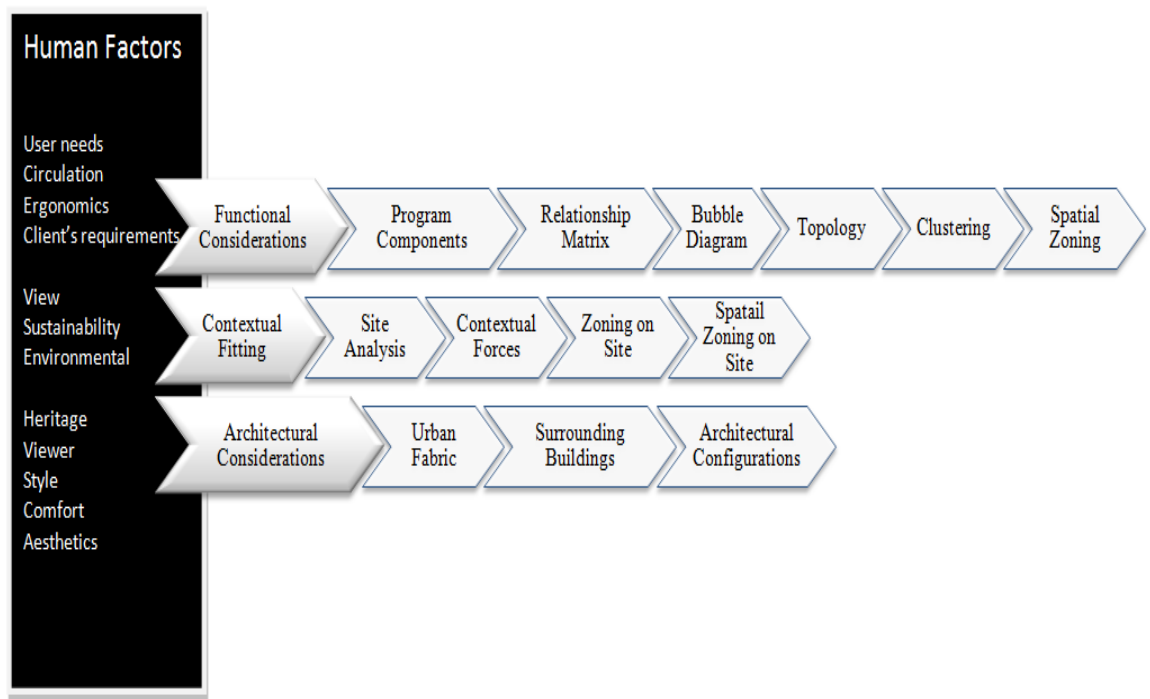

Figure 3: A Proposal for a Systematic Design Reasoning Process.

To customize the solution suggested by the functional template, the context of the given design is analyzed. This contextual fitting stage includes the investigation of the site, climate and other tangible and intangible contextual forces such as the physical, social and cultural environment as well as the geometric language of the surrounding urban components. The study of the external forces should personalize the abstract zoning that was produced by the functional solution stage. Such a personalization should produce a "spatial zoning on site" represented three-dimensionally on the actual site settings. Up to this point, the functional and the contextual impacts are taken into consideration, but the formalistic considerations are not yet considered. The urban fitting and the architectural identity of the surrounding buildings and outdoor spaces should be added at the third stage to transform the previously generated contextual spatial zoning diagram. Although the diagram of Fig. $\mathbf{3}$ is shown as a sequential development for organizational considerations, the order of the three phases can be traded off as needed. The sequence is in-deterministic and is proposed to organize the work but not to restrict it.

Considerations of the human factors of design in the functional solution phase are essential to the solution processing. Examples of these that are related to the functional component include: user needs, anthropometrics and ergonomics, client's and other building stakeholders' requirements, space flow, circulation and behavior patterns and interior design requirements. Examples of the context-related considerations include: view, acoustics, thermal comfort, orientation, sustainable solutions, 
environment-friendly considerations, accessibility, interactive elements and social aspects of users. Examples of aesthetics considerations include design formal issues, cultural reflection, architectural style, taste of internal and external users and viewers, and relationship to temporal, demographic and geographic factors.

4.2 Diagrams for Design Development. As a building may be considered a livable sculpture that relates to its environment, the design generation can be visualized as a three-dimensional chart the axes of which are function, context and form [see 24] (see Fig. 4). Along the function axis, the internal forces of the design problem at hand are considered. These include the program components as well as their interactions and relationships. The set of the external forces that may affect and be affected by the design represent the area around the context axis. The aesthetic considerations are represented by the area surrounding the third axis which stands for the form component of design. When isolating each projection of the three-dimensional chart a plane that represents the reciprocal relations between the two axes results (see Figs.5 and 6 for clarification). In the functionform plane, the circle closer to the function axis represents the space arrangement and planning part of the design development. The circle closer to the form axis represents the set of forms that are directly produced as a response to functional requirements.
When the area where the two circles overlap is developed it leads to the production of a working form for the design problem.

In the function-context plane, the circle closer to the function axis represents the space programming part of the design development. The circle closer to the context axis represents the set of contextual functions that are directly produced as a response to the contextual and functional requirements. When the area where the two circles overlap is developed it leads to the production of a spatial organization-onsite for the design problem.

In the context-form plane, the circle closer to the form axis represents the formal fitting decisions of whether to solve the design problem by contrast or harmony with the surroundings. The circle closer to the context axis represents the set of contextual forms that may affect the generated forms. When the area where the two circles overlap is developed, it leads to the production of a social form which represents how the individual form will interact with its community of surrounding forms. The areas surrounding each axis are illustrated in Fig. 5 and further illustrated in Fig. 6. The desired design is a development of the area where the three diagonal circles meet and where all requirements are considered. Central to the three axes is the human aspects related to each component. These include user behavioral and social patterns and their impacts on the design recommendations.

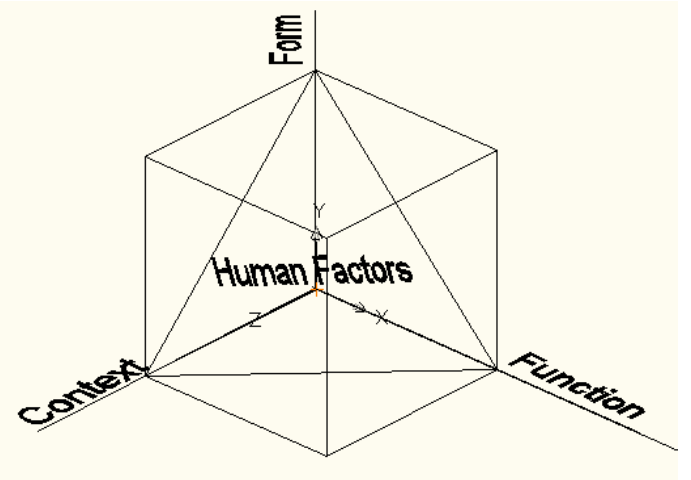

Figure 4. A 3D Chart of the Components of Architectural Design.

Each of the circles that are aligned with an axis describes a set of activities associated with that axis. For example, each of the two circles that touch the function axis in Figures 5 and $\mathbf{6}$ includes the study of the set of internal forces, such as the program development and components' internal relationships analysis; the space arrangement study; and the derivation of the abstracted functional solutions. All these activities are illustrated in Fig. 6. For the context circle, the set of the external forces are studied. It includes the analysis of the site, its climate and surrounding built and natural environment, and the contextual fitting solution which proposes a solution that is in harmony with its context, or one that adopts contrast to stands out in its environment (Fig. 5). In the form circle, the structural issues and their influences on form are studied. The principles of composition are also analyzed and applied to the given design problem. The design activities within this circle should end with an aesthetic or formalistic solution for the problem.

According to the chart illustrated in Figures 4, 5 and $\mathbf{6}$, the concept of 'form follows function' can be adjusted to 'form, function and context follow each 
other' and Le Corbusier's description of a house as ' a machine for living' can be adjusted to its characterization as 'a sub-machine that coordinates with others for living' to make it more responsive to actual design problem settings. Within this updated concept, decisions about form-making must be contextualized to consider its connections with the surroundings.

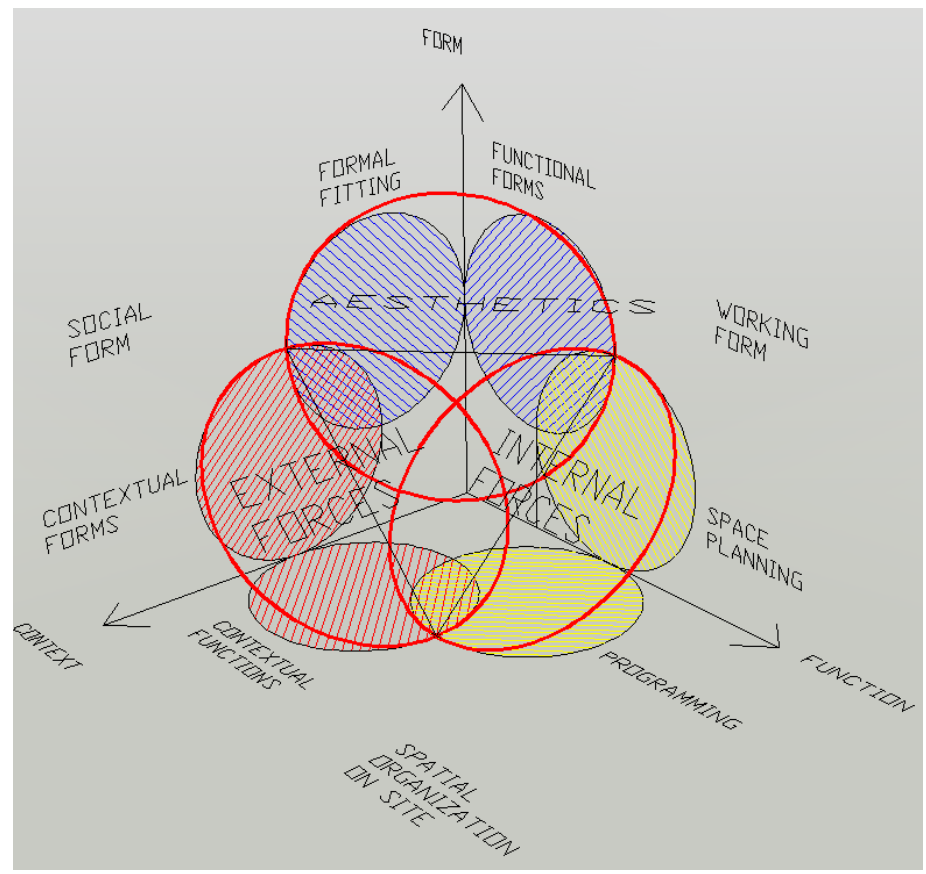

Figure 5. The Major Ingredients of Design Generation.

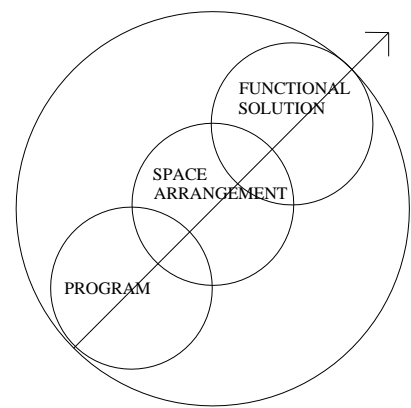

FUNCTION

Internal Forces

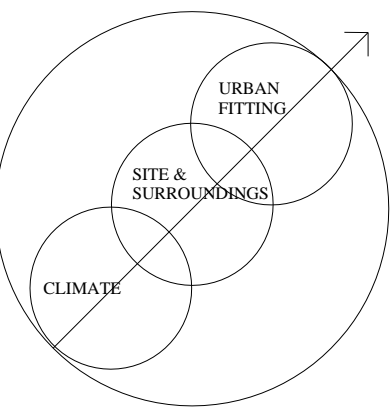

CONTEXT

External Forces

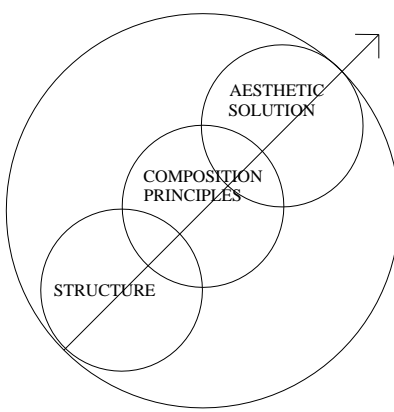

FORM

Aesthetics

Figure 6. The Structure and Sequence of Each Design Component.

Design may be viewed as an informed response to interplay between groups of forces. These include external, internal and aesthetic forces (Fig. 7). According to this view, forces in each category need to be listed and assigned a weight according to their impact. The interface between each pair of categories represents the area where design issues that belong to both categories should be considered. As a result of this diagram consideration, a design should balance various forces, prioritize them and optimize their impacts on the generated design.
Since form is the main interface through which all other design considerations are manifested and communicated to users, it will be the focus of discussion in the following section.

4.3 Diagrams for Design Assessment. Once a design product is documented and presented, it is possible to evaluate its satisfaction and compliance with the preset criteria and goals. It may also be examined by comparing it with other similar designs that are 
previously considered 'good' solutions. The design assessment can be carried out in three levels. The first

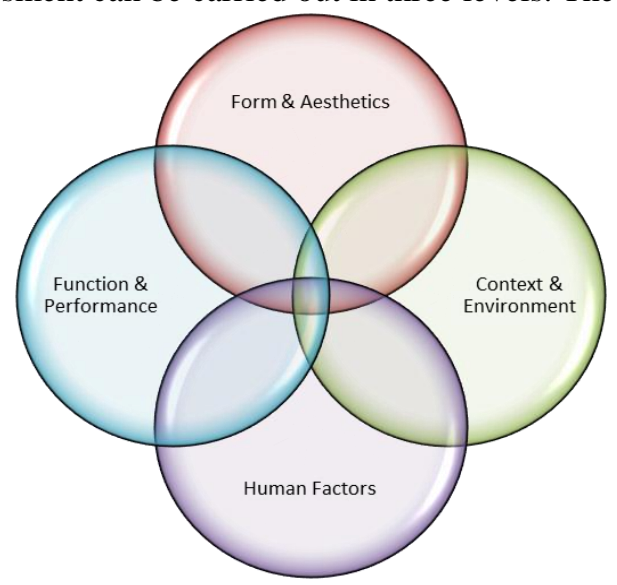

Figure 7. Design as an Interplay of forces.

and basic level of the evaluation is to check a design's compliance with the given codes and regulations to examine its safety and legality. The second level is the product quality assurance that goes beyond legal requirements to guarantee a pleasant experience for users and viewers. The third level evaluates the design at hand for its uniqueness, innovation and contribution to previous similar designs. At least within the first level, the physical structure and supportive systems should be examined for their stability and functioning. Similarly, a performance simulation can be conducted to predict the user's experience outside and inside the design product. The simulation can be conducted to evaluate the circulation flow, behavioral patterns and event-based simulation that can help the evaluator to imagine the design under daily, weekly, monthly or yearly scenarios or even under emergency circumstances. Furthermore, the evaluation can be extended to test the thermal and acoustics comfort level within the product and to evaluate its aesthetic attributes. The post-design evaluation components are illustrated in Fig 8.

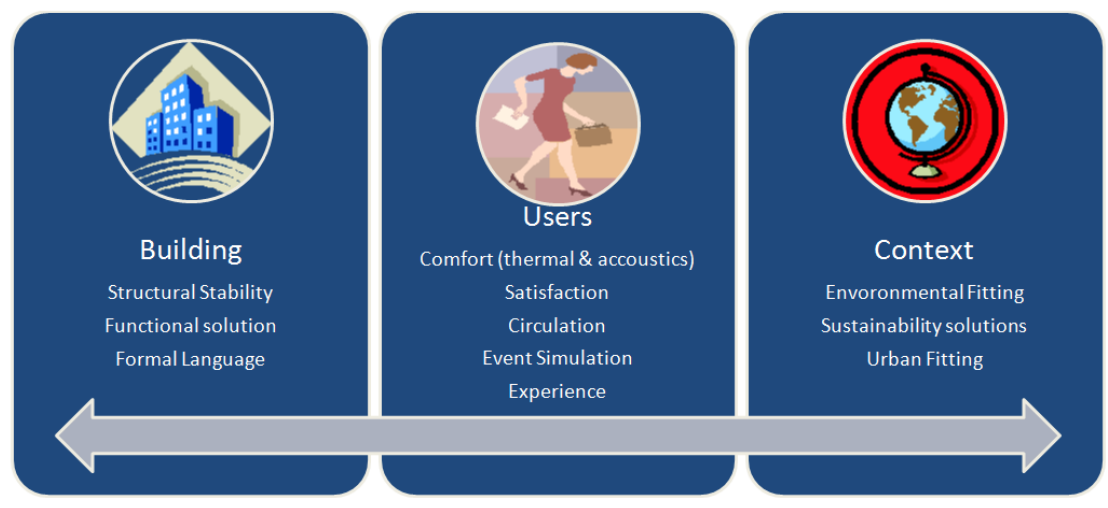

Figure 8: Post-Design Evaluation Diagram.

5. Form Architecture. It is possible to categorize the morphology of architectural form into two major levels. These are the planar and the spatial levels (Fig 9).

5.1 The Planar Ingredients of Form. The planar level includes the plan articulation and the facade treatment activities. In the plan articulation level, the major design activities include four major tasks. These are (1) the program component arrangement which requires decisions about zoning and topology of the components, (2) the contextual fit of the spatial layout which takes into consideration the design impact on its surroundings as well as the influence of the contextual forces on the design, (3) the opening distribution in the plan layout, and (4) the wall/partition assignment of the spaces' limits.

In the façade treatment level, three major design activities are considered. These include (1) decisions about the opening treatment such as window size, shape and location, and entrance location, shape, hierarchy and emphasis, (2) morphology of façade which includes the void and solid distribution, the internal and external proportions of the façade components, and their topological relationships, and (3) the surface treatment of the façade which includes issues such as color, texture, ornament, and use of false elevation.

5.2 The Spatial Considerations of Form. In the spatial level, there are three major design considerations. These include (1) massing methods, (2) clustering schema, and (3) enclosure treatment. The massing methods are strongly related to five major areas. These are: (1) the geometric language of the composition, (2) the decisions about whether to group the main masses using subtraction or addition, (3) the way to move the planar treatment from lines to planes, (4) the transition from two-dimensional planes 


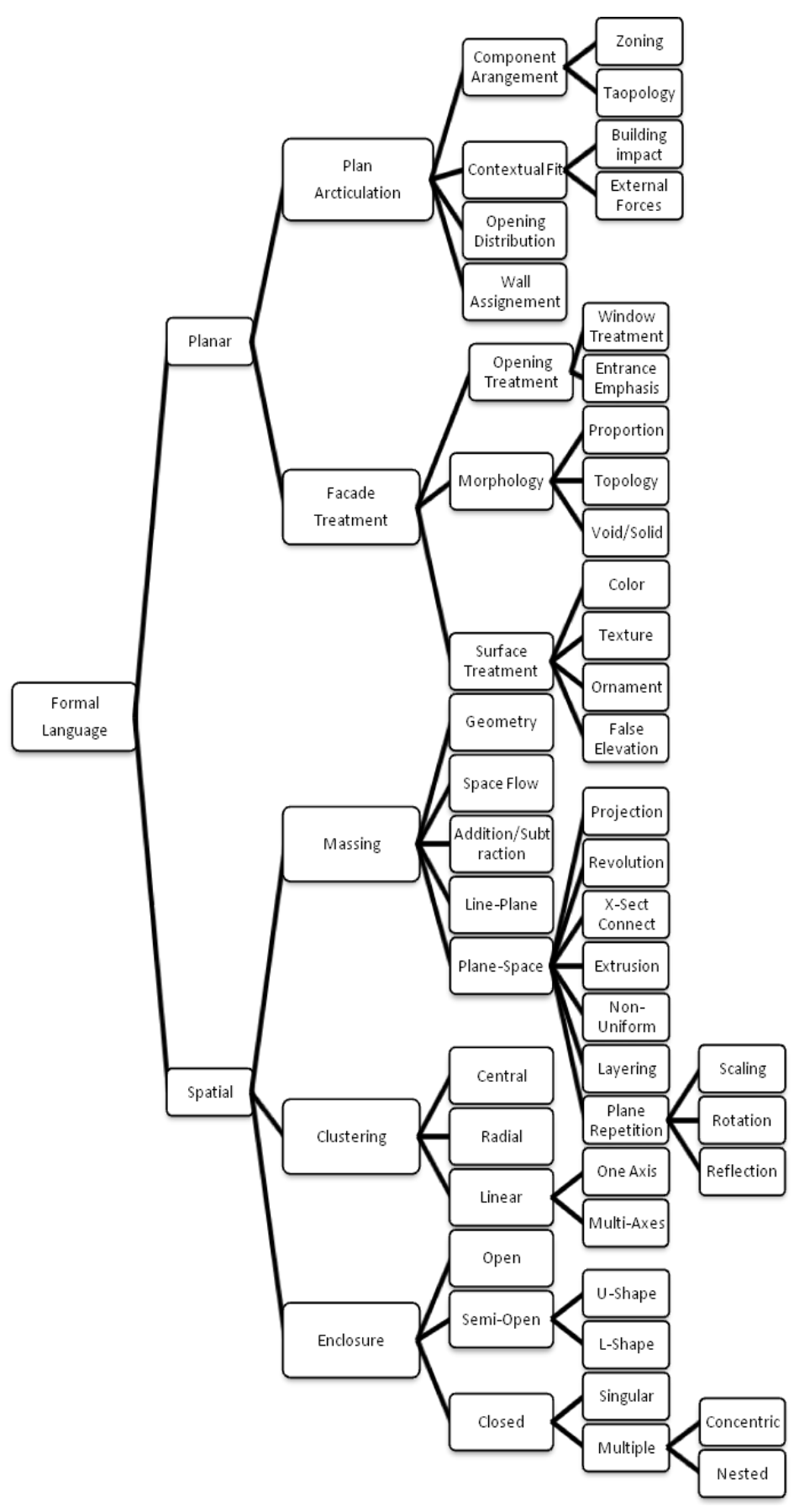

Figure 9: A Hierarchical Chart of Form Architecture.

into three-dimensional masses, and finally (5) the flow of spaces in the major and minor masses. The transformation of a plane into a space can be carried out using the most common method that is projection, or using revolution, extrusion along a path in space, connecting two cross-sections with a continuous surface, flat or interlocked layering of different planes in space, covering a space with a non-uniform surface, polyhedral transformation of faces, or plane repetition. This latter method can be conducted using translation of a plane in space along a path or axis optionally combined with scaling, rotating or reflecting each plane to vary it from the preceding ones.

The clustering of spaces can be carried out using the linear, central or radial schemes. The linear accumulation of masses can be executed vertically or horizontally through one axis or more.

The design masses can be grouped without a defined enclosure or with closed or semi-open ones. The closed enclosure, in turn can be achieved by a singular court or multiple concentric or nested outdoor spaces. The semi-open enclosure can be achieved by grouping masses around a court that is open from one side (Ushaped) or two sides (L-shaped).

In a systematic design methodology, a design may consider all these levels and sublevels for the generation of the final form of a physical design. Such a consideration helps guarantee a minimum level of satisfactory design in which major volumetric and planar articulation techniques are at least taken into consideration.

\section{A Diagram-Aided Design Project: Implementation and Discussion}

As stated earlier, all five phases; input, process, output, presentation and assessment; are necessary for design processing. The proposed diagrams are expected to be integrated and cycled through during all phases to conduct a systematic design process. The goal of their implementation is to guide design sequence but not to restrict it. The combination of the diagrams of the five major components of designing phases forms a basis for a diagram-aided approach to design processing in its various phases. Using the diagrams illustrated in the previous sections is suggested to help designers externalize their thinking process and to help learners apply a structured method that may guide them through design knowledge building and product development.

It is argued that applying the proposed approach to designing helps designers to organize their thoughts, guides them through design processing and develops their evaluative skills. In addition, it is suggested that the approach may help 
novice designers to learn about design building and development. To test the validity of these arguments, the diagrams illustrated so far were introduced and explained to architectural design students. The students of third year school of architecture in Jordan University of Science and Technology were assigned a design problem and asked to apply the diagrams as guidelines in their various processes. The student group included 21 students in a real design studio setting. The design problem was about designing a seaside resort hotel that is located in Aqaba port in Jordan. After applying the diagrams as conceptual and operative tools, the students were asked to provide their experiment feedbacks through informal discussions, jury presentations and structured anonymous questionnaire. The questionnaire was conducted after the term in which the design project was assigned ended to guarantee more objectivity in the responses. The questions in the questionnaire were designed to evaluate students' deep learning reflections in addition to their evaluation of the diagram-aided approach. Questions about deep learning competencies were structured to emphasize higher-order, integration and reflection in participant students' learning abilities [25-27]. To test participants' higher-order learning, they were asked if applying the diagrams for design processing help them connect the design problem assigned to them to real life practice situation (Q1), and if it makes them think if the design methods they learn would be useful for them after graduation $(\mathrm{Q} 2)$.

To assess the integration factor, some questions were added to examine if using the proposed diagrams encourage students to relate what they have learnt in design studio to other theoretical courses (Q6), and connect their knowledge to other practical courses (Q6). The impact of the diagrammatic approach on enhancing students' reflections was examined through questions about whether implementing this approach remind participants of materials they already know from other resources and of experiences they have had lived (Q6), and if the approach helps them restore previous images in new lights (Q5).

The questionnaire also tested the impact of the diagrammatic approach on students' achievements. These were measured by questions about the approach's enhancement of design process organization (Q7, Q8, and Q10), product optimization (Q9), evaluation skills (Q12) and understanding of new layers and meanings underlying design process (Q11).

The questions asked are:

Does applying the diagram-aided approach to solve design problems:

1. Help you connect the design problem to real life architectural practice?
2. Make you think that you may apply the approach in professional practice after graduation?

3. Encourage you to relate what you have learnt in design studio to other theoretical courses?

4. Encourage you to apply similar approaches in other practical courses?

5. Remind you of materials and experiences you already know from other resources?

6. Help you restore previous knowledge and images in new ways?

7. Help you organize your pre-design sequence?

8. Help you start the design solution process?

9. Make you produce a more balanced design product?

10. Make you move between the different design stages more smoothly?

11. Emphasize new layers of design forces you usually ignore?

12. Develop your evaluation skills?

Students were asked to answer each question by selecting one of these options:

$\square$ Strongly agree (10 points)

$\square$ Agree (7 points)

$\square$ Sometimes (3 points)

$\square$ Don't agree (0)

The number associated with each answer is the numerical score (out of 10) correspondent to each option.

All questions in the survey were based on students' comparison between the diagrammatic approach and the previous trial/error conventional one. Questions from 7-12, in particular, emphasize this comparison.

Students' responses to the questionnaire are illustrated in Fig. 10. In Fig. 10, the students' answers are represented by a Radar chart in which each continuous poly-line represents all answers given by all students to one question. The average score given by all students to each question is illustrated in Fig. 11.

As Figs. 10 and 11 show, students' responses for each question vary in their ranges (lower and higher ends), but common grounds for the majority of answers for each question can be found. These are represented by the average score of each question (Fig. 12). As Fig. 11 shows, the participants reported positive reactions about the diagrammatic approach enhancement of their achievements. The highest scores were given to Q7 (impact on the organization of pre-design sequence), followed by Q12 (the approach's contribution to developing evaluative skills). The approach also seems to help emphasizing new layers of design forces students usually ignore (Q11), to produce a more balanced design 


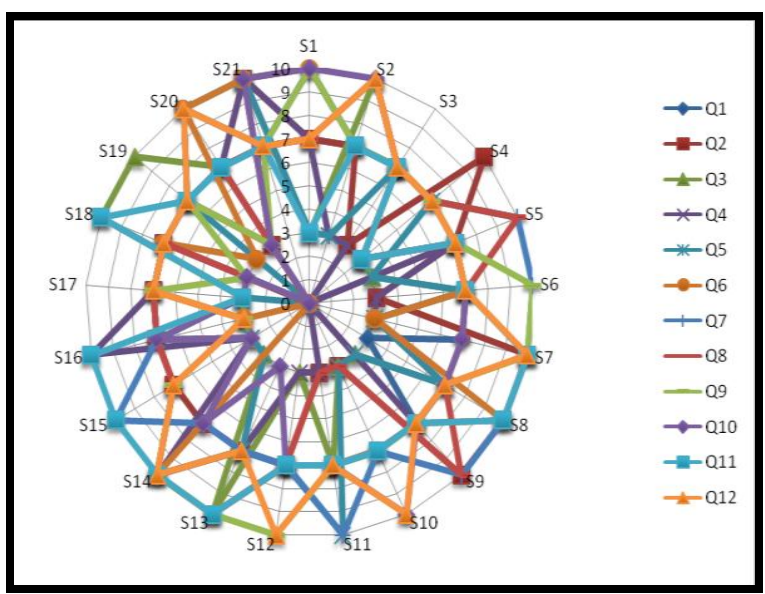

Figure 10. Students' answers to structured questionnaire.

product (Q9), and to aid in initiating the design process $(\mathrm{Q} 8)$. Less positive responses were given to the diagrams' role in smoothing transition between various stages (Q10). Moreover, the approach seems to improve the deep learning abilities of the students. It helps encourage learning by reflection (Q5 and Q6) and integration between learned topics (Q3 and Q4). Among these, the order from higher to lower score is given to higher-order learning, then reflection, followed by integration. The scores of the achievement measures are higher than those of deeplearning enhancement. As a result, the approach seems promising in helping students process their design but needs more development to more significantly enhance deep-learning skills in general, and issues of various topic integration in particular.

As illustrated in Fig. 11, responses to questions 712 support the application of the diagrammatic approach as opposed to the conventional one. In

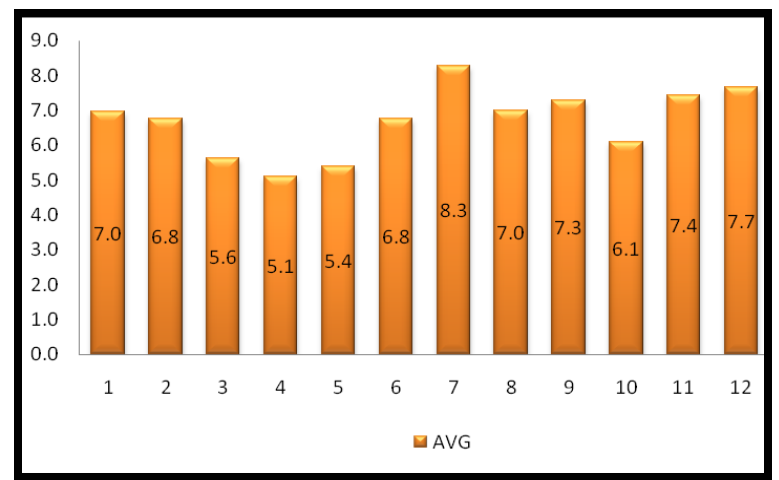

Figure 11. Average score of the answers of each question in the Diagrammatic Approach.

addition, feedback from the project evaluators and jury members emphasized that the projects reflected deeper thinking than they used to see in other design products for the same students.

Furthermore, in order to examine the impact of the diagram-aided approach in design processing, a comparison was made between the group of students who applied it and another control group. The same project was given to another section of 19 third year Architectural students in the same college. The approach they used was the trial/error where they develop a design proposal based on initial analytical stage. Then, based on instructors' and jury members' feedback, they modify the solution. Similar to the other section, after the semester ended, students were asked anonymously to respond to the same questionnaire. A comparison between the average scores for each question is illustrated in Fig.12.

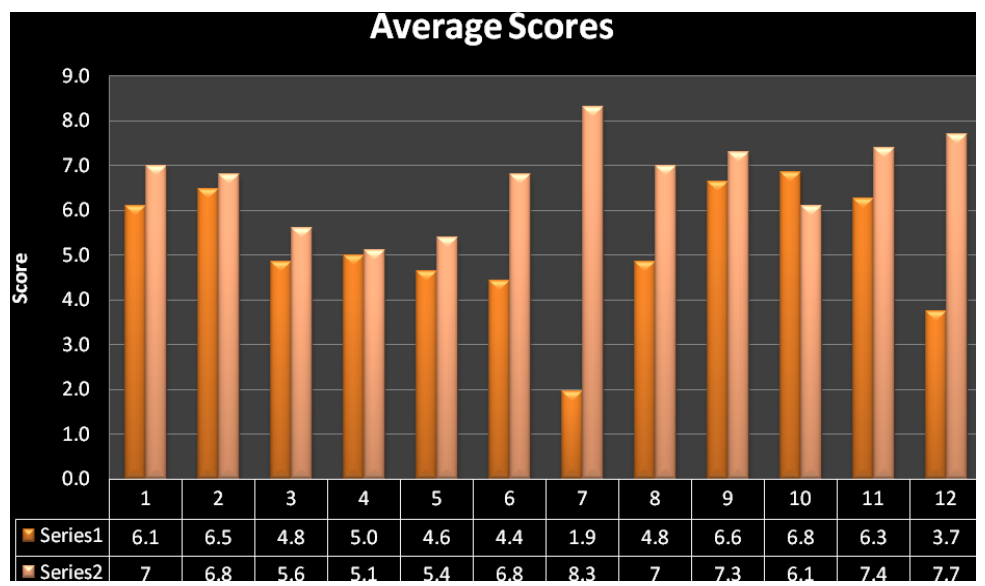

Series 1 (dark bar): Trial/Error design development process responses

Series 2 (light bar): Diagrammatic approach design development process responses

Figure 12. A Comparative chart for survey responses to using two different design approaches. 
In Fig.12, Series 1 (dark bar) represents students' responses to questionnaire about the Trial/Error design development approach processing, and Series 2 (light bar) represents the responses for the Diagrammatic approach design development process. As Fig. 12 demonstrates, the proposed approach seems to help in the higher learning aspects (where it helps connect knowledge to real life settings), and in reflection (where it helps connect design to other courses and restore images from previous experiences). It also seems to significantly help design process organization and students' self evaluation. However, the proposed approach seems less helpful in the smooth transition between different design processing phases (Q10).Despite its limitation, the proposed approach may contribute to scholarly efforts in knowledge recycling of design processing [e.g. 2832], which, in turn, represents an area of research where future extensions of this article may build on.

\section{Conclusion}

Diagrams represent significant design aid tools in the toolbox of designers. There exist an extremely limited number of design analysis and synthesis diagrams. Added to this deficiency is that the known ones are slowly -if ever- developing in most design areas. Although diagrams help in the visualization of designing in many knowledge areas, the scope of this paper is focused on the architectural design domain. In this paper, a set of operative and figurative diagrams are proposed to help organize and inform the design input and reasoning, processing, production and assessment activities. Due to the special importance of form in architectural design, additional diagrams for form generation are introduced and discussed through a design project that is assigned to architectural students. The contribution of the diagrams as catalysts for systematic design processing is tested in real design studio settings. The results of the applicability testing of the proposed diagrams support its positive impact on improving users' achievement and their learning skills. Future extensions of this paper include more testing in different practical and educational settings. Another extension is that the diagrams can be further developed into computer-aided design guides that may vary in their sequences according to designers' input.

\section{References:}

[1] Goel V (1995) Sketches of Thought. Cambridge: MIT Press

[2] Clayton M J (2000) Diagramming aesthetics: modernism and architecture in the $21^{\text {st }}$ century, in M Anderson, P Cheng and V Haarslev (eds), theory and application of diagrams: first international conference, diagrams 2000
(Edinburgh, Scotland, 2000) New York: Springer, pp 257-270

[3] Suwa M, Gero J and Purcell T (2000) Unexpected discoveries and S-invention of design requirements: important vehicles for a design process, Design Studies, 21(6), pp 539-567

[4] Vidler A (2000) Diagrams of diagrams: architectural abstraction and modern representation, Representations (72), pp 1-20

[5] Stouffs R (2001) Visualizing information structures and its impact on project teams: an information architecture for the virtual AEC company, Building Research and Information 29 (3), pp 218-232

[6] Ching F (2003) Architectural Graphics (4 ${ }^{\text {th }}$ ed) New York: John Wiley

[7] Vande Moere A (2005) Form follows data: the symbiosis between design and information visualization, in Proceedings of CAADfutures, (Vienna, Austria, 2005) Okk Verlag, pp.31-40

[8] Do E and Gross M D (2001) thinking with diagrams in architectural design, Artificial Intelligence, 15(1-2), pp 135-149

[9] Knoespel K J (2002) Diagrammatic transformation of architectural space, Philosophica 2002: Diagrams and the Anthropology of space, 69, pp 11-36

[10] Cheng P, Lowe R, and Scaife M (2001) Cognitive science approaches to understanding diagrammatic representations, Artificial Intelligence Review, 15(1-2), pp 79-94

[11] Shimojima A (2001) The graphic-linguistic distinction: exploring alternatives, Artificial Intelligence Review, 15(1-2), pp 95-114

[12] Stenning K and Lemon O (2001) Aligning logical and psychological perspectives on diagrammatic reasoning, Artificial Intelligence Review, 15(1-2), pp 29-62

[13] Bauer M I and Johnson-Laird P N (1993) How diagrams can improve reasoning, Psychological Sciences, 4 (6), pp 372-378

[14] Chandrasekaran B, Glasgow J and Narayanan N $\mathrm{H}$ (Eds) (1995) Diagrammatic Reasoning: Cognitive and Computational Perspectives, Cambridge: MIT Press

[15] White, E T (1983) Site Analysis: Diagramming Information for Architectural Design, Tucson, Arizona: architectural media ltd

[16] Alexander C (1966) Notes on the Synthesis of Form, Cambridge: Harvard University Press

[17] Laseau P (1986) Graphic Problem Solving for Architects and Builders ( $2^{\text {nd }}$ ed) Boston: Van Nostrand Reinhold 
[18] Lockard W K (1977) Drawing as a Means to Architecture, Tucson: Pepper Publishing

[19] Dorst K and Cross N (2001) Creativity in the design process: co-evolution of problem solution, DesignStudies, 22(5), pp 425-437

[20] Ervin S M (1990) Designing with diagrams: a role for computing in design education and exploration, in M McCullough, W J Mitchell and P Purcell (Eds), The Electronic Design Studio: Architectural Knowledge and Media in the Computer Era, Cambridge: MIT Press, pp $107-$ 122

[21] Eilouti B H (2005) The representation of design sequence by three-dimensional finite state automata, in D. Zinn (Ed), The International Institute of Informatics and Systemics(Orlando, Florida, USA, 2005), pp. 273-277

[22] Eilouti B H (2007) “A Spatial Development of a String Processing Tool for Encoding Architectural Design Processing”, Art, Design and Communication in Higher Education, Vol. 6, No. 1, pp. 57-71

[23] Eilouti B H, and Vakalo E G (1999) Finite state automata as form-generation and visualization tools, in E. Banissi and F. Khosrowshahi (Eds.), Information Visualization IV 99, Los Alamitos: IEEE Computer Society, pp. 220-224

[24] Eilouti, Buthayna H (2010) Diagrams as Design Catalysts, Design Principles and Practices: An International Journal, Common Ground Publishers, 4 (2) pp 217-231
[25] Beatie, V., Collins, B., and McInnes, B. (1997) Deep and surface learning: A simple or simplistic dichotomy? Accounting Education, 6(1), pp 1-12

[26] Biggs, J.B. (1989) Approaches to the enhancement of tertiary teaching. Higher Education Research and Development, 8, pp 7-25

[27] Tagg, J. (2003) The learning paradigm college. Boston, MA: Anker.

[28] Eilouti, Buthayna H. (2009) Design Knowledge Recycling Using Precedent-Based Analysis and Synthesis Models, Design Studies, Elsevier, Vol 30, No.4, pp. 340-368

[29] Eilouti, Buthayna H. (2011) Environmental Knowledge in Engineering Design Processing, The 5th International Conference on Knowledge Generation, Communication and Management: KGCM 2011, pp. 370-375, Orlando, Florida, USA

[30] Eilouti, Buthayna H. (2009) Knowledge Modeling and Processing in Architectural Design, The 3rd International Conference on Knowledge Generation, Communication and Management: KGCM 2009 , Orlando, Florida, USA

[31] Eilouti, Buthayna H. (2007) Models for the Management of Precedent-Based Information in Engineering Design, in D. Zinn (ed), The International Institute of Informatics and Systemics WMSCI2007, pp. 321-326, Orlando, Florida, USA

[32] Eilouti, B. H. (2012) Knowledge Recycling and Transformation in Design, New Research on Knowledge Management Models and Methods, ISBN 978-953-51-0190-1 , pp. 65-76, INTECH 